\title{
Criminologie
}

\section{De l'esprit et de l'application des normes}

\section{Alice Parizeau}

Volume 11, numéro 2, 1978

Normes et politique criminelle

URI : https://id.erudit.org/iderudit/017090ar

DOI : https://doi.org/10.7202/017090ar

Aller au sommaire du numéro

Éditeur(s)

Les Presses de l'Université de Montréal

ISSN

0316-0041 (imprimé)

1492-1367 (numérique)

Découvrir la revue

Citer cet article

Parizeau, A. (1978). De l'esprit et de l'application des normes. Criminologie, 11(2), 25-41. https://doi.org/10.7202/017090ar d'utilisation que vous pouvez consulter en ligne.

https://apropos.erudit.org/fr/usagers/politique-dutilisation/ 
Dans le contexte nord-américain les commissions d'études et d'enquêtes, modelées selon la tradition britannique, ont toujours occupé une place importante dans le domaine des réformes de la justice. Au lieu d'imposer les changements, les autorités font appel ainsi aux spécialistes, tout en se réservant la possibilité de décider de l'application des propositions.

Il n'en reste pas moins que les études menées en matière du droit criminel et de son application furent particulièrement importantes au cours des dix dernières années autant au Canada ${ }^{1}$ qu'aux États-Unis ${ }^{2}$.

C'est dans ce cadre que s'inscrivent celles entreprises par l'Association du Barreau américain. En effet, dès 1964, l'Association a décidé de créer une Commission chargée d'élaborer des normes de conduite et des règles de pratique et de les soumettre à la Cour suprême des États-Unis en lui demandant son approbation. Actuellement, c'est-à-dire quatorze ans après, le travail est terminé et publié sous forme d'une série de dix-huit volumes qui représentent le rapport final de divers comités chargés d'examiner des secteurs spécifiques.

Sans vouloir présenter cette étude comme un modèle applicable éventuellement dans le contexte québécois, il nous semble intéressant néanmoins d'offrir à nos lecteurs un aperçu des recommandations qu'elle contient. Afin de la rendre aussi claire que possible, nous la présentons en suivant le plan adopté par la Commission et nous nous efforçons de donner un résumé schématique de chacun des chapitres. Étant donné, toutefois, qu'il s'agit d'un travail énorme auquel ont collaboré les meilleurs juristes, professeurs et avocats américains, nous suggérons à ceux qui sont

1. A cet égard il est important de mentionner, entre autres, pour le Canada, les travaux de la Commission Ouimet. Canada, Ministère du Solliciteur général, Rapport du Comité canadien de la Réforme pénale et correctionnelle, Ottawa, Information Canada, 1969, 554 p.

2. Aux Etats-Unis, il convient de souligner surtout l'apport des recherches effectuées par la Commission Katzenbach. U.S.A., Commission on Law Enforcement and Administration of Justice, $A$ Report by the President's Commission on Law Enforcement and Administration of Justice, Washington (D.C.), 1967. 
intéressés de référer aux volumes pertinents dont nous indiquons la liste à la fin de cet article.

L'ensemble des normes sélectionnées par la Commission peut être divisé en trois catégories, soit les règles qu'on peut qualifier d'administratives s'appliquant entre autres à la police, celles concernant l'éthique professionnelle et celles qui ont trait aux normes de l'application de la justice criminelle.

\section{Les règles administratives - les services policiers}

Le premier chapitre du rapport général de la Commission concerne les services policiers. Comme tous les autres chapitres du rapport, il comprend un plan logique, dont la première partie vise à expliquer les raisons pour lesquelles l'élaboration des normes semble indispensable et la deuxième, les conclusions et l'énumération des règles de base. Or, il est intéressant de noter que les objectifs sélectionnés par les auteurs de l'étude américaine comme les plus importants correspondent à ceux invoqués le plus fréquemment au Québec. En d'autres termes, des deux côtés de la frontière les difficultés apparaissent comme sensiblement similaires.

C'est ainsi que les commissaires constatent qu'il est indispensable d'améliorer la qualité des services de la police et que cette tâche est d'autant plus difficile que les exigences qu'on manifeste à son égard sont souvent irréalistes sinon contradictoires.

Parallèlement, on insiste sur le pouvoir réel de la police. Un agent qui est en train de travailler sur une patrouille, écrivent les commissaires, prend plus de décisions discrétionnaires qui affectent directement la vie des citoyens qu'un juge. Or, un magistrat peut baser sa sentence sur des textes législatifs, tandis qu'aucun guide ne saurait probablement être utilisé par le policier. Il ne peut se fier qu'à son expérience et qu'à son bon jugement, dont la définition même est parfaitement imprécise et peut varier drastiquement selon les individus.

Un ensemble de normes doit viser à combler partiellement les conséquences de ce genre de disparités, mais ne peut dans aucun cas les éliminer complètement.

En deuxième lieu, la police est critiquée non seulement par le public, mais aussi par les professionnels qui sont en contact 
avec elle, dont en particulier les procureurs et les avocats. En fait, il devrait s'agir là de diverses formes de collaboration, mais en pratique on constate plutôt un ensemble d'attitudes négatives. À cet égard, il est bien malaisé de prétendre, comme le font certains juristes, que la police n'est pas faite pour avoir ses propres politiques d'action, car quoi qu'on fasse, elle les conçoit et elle les applique au jour le jour.

Selon le juge en chef américain, Warren E. Burger, les deux dimensions principales des réformes qui s'imposent comprennent, d'une part, une meilleure formation et sélection des officiers de police et, d'autre part, un cadre élémentaire de normes d'action. Ce cadre découle en quelque sorte de deux principes fondamentaux.

Tout d'abord, puisque la police doit assumer la poursuite des criminels, il convient d'améliorer constamment son efficacité. Il faut reconnaître également que cette efficacité dépend, par ailleurs, de plusieurs services qui font partie du système de la justice criminelle ou se situent à l'extérieur de son cadre et que, dès lors, il est indispensable d'assurer entre eux des liens et des moyens de collaboration.

On ne saurait, en outre, obtenir le rendement maximal des services de la police sans tenir compte de la multiplicité des tâches et des objectifs qui lui sont confiés. Lidentification et l'arrestation des personnes coupables de divers délits ne constituent à cet égard qu'une facette du rôle de la police, les autres étant l'aide aux citoyens, la protection de leurs droits, l'intervention dans certains types de conflits et la participation, avec d'autres services, dans les diverses interventions visant à assurer la prévention de la criminalité.

L'élaboration des normes doit tenir compte du fait que la diversité des rôles de la police ne peut que s'accentuer à l'avenir. Après les avoir identifiés et évalués, il faut dégager, par conséquent, une échelle des objectifs, classés selon leurs priorités. Å cette étape s'imposent des précisions relatives aux méthodes devant être appliquées, aux coûts de leur mise en pratique et aux nécessités des programmes expérimentaux et d'évaluation susceptibles de confirmer ou d'infirmer les cadres théoriques.

Il nous est impossible ici de reproduire les normes proposées par la Commission car il s'agirait alors d'une énumération 
fastidieuse et de lecture difficile ; nous nous contentons donc uniquement de résumer, en ce qui a trait aux études sur la police, leurs principaux thèmes.

Sous le titre général des objectifs et des priorités des services policiers, la Commission a dégagé les facteurs qui interviennent dans leur réalisation, les responsabilités qu'ils impliquent, ainsi que les critères d'action des divers niveaux de police : locale, régionale et fédérale.

A titre d'exemple, nous reproduisons, ci-dessous, certains de ces facteurs dont :

(i) l'étendue du mandat législatif donné à la police ;

(ii) l'autorité de la police à user de la force ;

(iii) la capacité de la police à faire enquête ;

(iv) la disponibilité continue de la police vingt-quatre heures sur vingt-quatre ;

(v) la pression de la communauté sur les services de la police.

En ce qui a trait aux responsabilités de la police, la Commission indique, entre autres, la liste schématique suivante :

(i) l'identification des criminels et des activités criminelles, leur contrôle et la présentation de la preuve devant les cours ;

(ii) réduction des possibilités de commission de certaines actions criminelles par des différentes mesures, dont les patrouilles entre autres ;

(iii) aide apportée aux individus en danger ;

(iv) protection des droits garantis par la constitution ;

(v) organisation de la circulation des véhicules automobiles ;

(vi) aide aux personnes incapables de fonctionner seules (handicapées, invalides, etc.) ;

(vii) intervention en vue de régler certains conflits familiaux ou autres ;

(viii) identification des problèmes occasionnés par l'application de la loi, ou des règlements gouvernementaux ;

(ix) la création et le maintien dans la communauté d'un sentiment de sécurité ;

(x) l'organisation et la protection de l'ordre civique ;

(xi) la disponibilité des divers autres services d'urgence. 
L'énumération indiquée plus haut est une traduction non littérale du texte extrait du rapport de la Commission. Nous le citons afin de donner une idée de la tentative de classement et de sélection entreprise par la Commission.

Comme on le constate, il ne s'agit pas là d'une innovation mais plutôt d'une analyse logique et détaillée de l'action de la police.

La Commission a dégagé également plusieurs principes de base de cette action en insistant en même temps sur les moyens nécessaires à leur mise en pratique. Il convient de souligner également la façon exhaustive selon laquelle chaque sujet est traité et le fait que les Commissaires examinent en détail des problèmes aussi controversés que la syndicalisation des forces policières et leurs droits à des actions collectives. Certes, il ne faut pas considérer cela comme une innovation majeure puisqu'on relève dans le rapport les mêmes doutes et hésitations en ce qui a trait, par exemple, aux possibilités de grève de la police, que ceux qu'on trouve dans d'autres travaux effectués au Québec ou au Canada, mais il importe de souligner la clarté des prises de position ${ }^{3}$.

C'est ainsi que les Commissaires établissent des normes et des limites, en les justifiant par la nécessité de l'objectivité de la police et de sa disponibilité, qui excluent, à toutes fins pratiques, l'éventualité d'une forme de syndicalisation classique ayant pour arme ultime le refus d'exécuter le travail, soit la grève.

Contrairement aux spéculations philosophiques dont on abreuve les lecteurs dans certains rapports, celui de l'Association du Barreau américain est rédigé, en somme, de façon factuelle visant principalement à servir de document immédiatement utilisable pour l'élaboration de certaines réformes.

Le rapport traite de la même façon des divers autres services et organismes tels que l'Aide juridique, par exemple, mais on y étudie aussi en profondeur les normes devant s'appliquer à certains moyens d'action aussi controversés que l'écoute électronique entre autres.

3. Pour le Québec, voir en particulier : Jérôme Choquette, c.r., ministre de la Justice, la Justice contemporaine, Québec, Editeur officiel du Québec, avril 1975, 360 p. 
Selon le même modèle que celui que nous avons indiqué en analysant le volume sur les services de la police, les Commissaires établissent les facteurs justifiant l'écoute électronique, l'autorisant et la rendant indispensable mais en même temps, ils relèvent les limites dans lesquelles elle peut être appliquée. Ces limites comprennent tout aussi bien les catégories de délits concernés que le genre de criminels, les délais qui peuvent être accordés à l'action policière dans ce domaine et les normes régissant l'utilisation et la destruction, la confidentialité et la transcription des enregistrements ainsi obtenus.

Comparativement aux énoncés contenus dans les règlements et les législations, le texte du rapport qui porte sur l'action policière dans le domaine de l'écoute électronique, est à la fois descriptif, analytique et limitatif. Les normes servent dans ce sens non pas à préciser les pouvoirs de la police ou les droits des citoyens mais bien davantage à faire une synthèse entre les éléments de l'enquête, ses aspects techniques, les contraintes de la loi et les modalités empiriques de rédaction des comptes rendus. Le fait qu'au lieu d'un texte descriptif, on soumet tous les éléments en points précis, autorise des comparaisons avec d'autres systèmes et une acceptation, ou une remise en question, non seulement des législations, mais également, sinon surtout, des modes de leur application par la police et par tous les services concernés.

2. Les principes d'éthique professionnelle; le rôle de la poursuite, de la défense et des juges

Autant il est relativement simple, cependant, de dégager des normes d'action policière, telles qu'elles devraient être imposées, compte tenu des réalités actuelles, autant il est infiniment plus malaisé de préciser le même cadre de base en ce qui a trait à l'action des avocats. En effet, il ne s'agit pas là, uniquement, des règles professionnelles de pratique, bien connues des spécialistes en la matière, mais d'une analyse détaillée de toutes les relations qui s'établissent entre l'avocat, le client, le juge ou le jury et des règles qui doivent être adoptées à travers l'ensemble du processus en ce qui a trait à l'intervention d'un procureur et d'un défenseur.

Les auteurs du rapport admettent qu'ils n'apportent aucun élément nouveau mais qu'ils se sont efforcés uniquement de trans- 
crire ce qui se pratique et de dégager, à partir de là, des lignes de conduite devant être adoptées. Afin d'y parvenir et d'éviter d'être accusés d'autoritarisme, les Commissaires ont procédé à une très large consultation auprès des juristes à l'aide des entrevues et de l'application des questionnaires. Travail d'autant plus énorme qu'à travers les rencontres et les consultations faites lors de réunions de divers groupes et comités, on a voulu recenser et opérationnaliser la description d'une tradition judiciaire vieille de quatre cents ans.

Les résultats de l'étude comprennent trois dimensions.

Tout d'abord une approche plus théorique en ce qui a trait au système accusatoire et inquisitoire et la place du procureur et du défenseur dans ces deux cadres.

En deuxième lieu, on décrit les fonctions et les relations des avocats de l'accusation autant avec les prévenus, qu'avec les experts, les témoins, les juges, le jury et la police. On précise parallèlement les normes et leurs objectifs à toutes les étapes du processus de la justice criminelle lors desquelles le procureur est appelé à intervenir.

En troisième lieu, enfin, on traite de façon tout aussi détaillée de l'action de l'avocat de la défense.

Pourquoi un tel travail a été entrepris au niveau des professionnels qui, après tout, sont soumis, par définition, aux règles de pratique, décrites et appliquées par des organismes professionnels, tel le Barreau, et plus ou moins sévèrement contrôlées selon les divers contextes nationaux ?

A cette question, on peut fournir plusieurs réponses.

Selon les commissaires, il convient d'insister sur l'évolution qui a marqué, au cours des trente dernières années, l'ensemble du système de l'application de la justice criminelle. Plus récemment, des réformes liées à la déjudiciarisation ${ }^{4}$, par exemple, ont imposé un poids additionnel de responsabilités aux procureurs, mais aussi aux avocats de la défense. Contrairement au principe traditionnel de l'établissement de culpabilité, devant primer sur les décisions ultérieures relatives à la punition, ou au traitement,

4. Pour le Canada, voir à ce propos les travaux de la Commission de réforme du droit, document de travail no 7, la Déjudiciarisation, Ed. Information Canada, Ottawa, 1974, 30 p. 
on se préoccupe dans certains cas d'évaluer en priorité la personnalité du délinquant et sa capacité de réinsertion sociale. Aucun texte, aucune règle ne permettent au procureur de disposer d'une base vraiment incontestable susceptible d'appuyer ou d'infirmer sa décision. Les règles auxquelles il obéit sont des lois infiniment souples et individualisées. La tentative de lui fournir des normes de conduite représente à cet égard une innovation.

L'avocat de la défense, pour sa part, cesse de plus en plus fréquemment d'être rémunéré pour son travail par son client. Dans le domaine de la justice criminelle, au Canada comme aux Etats-Unis, la majorité des provinces et des états ont élaboré des services d'aide juridique. Ils sont accessibles, en principe, à ceux qui ne disposent pas d'un revenu, ou d'une fortune suffisante pour assumer la charge des honoraires d'un avocat, mais en pratique, dans le domaine de la justice criminelle, il est infiniment difficile d'évaluer la capacité financière des prévenus comme celle d'ailleurs des victimes. Exception faite des causes qui impliquent des compagnies, l'individu délinquant a rarement un salaire, ou un revenu officiellement déclarable, tandis que la majorité des victimes n'est pas en mesure ou ne désire pas entreprendre personnellement des poursuites.

La gratuité des services de la défense entraîne cependant des problèmes multiples liés à la liberté du choix de l'avocat et aussi au statut de ce dernier en tant que praticien ayant son bureau privé ou, comme salarié, employé par le Service de l'aide juridique.

Les normes élaborées par les commissaires traitent de tous ces problèmes-là et comme elles proposent des règles de pratique, basées autant sur celles qui existent dans les textes que sur l'expérience empirique, elles constituent un outil fort intéressant.

L'analyse de l'action des principaux agents du système de la justice criminelle se poursuit ensuite à travers celle des juges. Les auteurs du rapport ont rédigé, à cet égard, des normes ayant trait tout aussi bien à l'émission du mandat, qu'à l'ensemble des procédures qui comprennent l'établissement de la culpabilité et le processus de prise de décision, puis de l'exécution de la sentence.

Parallèlement, on présente des propositions relatives aux normes régissant la suspension, ou la mise à la retraite anticipée 
des magistrats qui se sont rendu coupables d'une conduite inappropriée, de négligence, d'inefficacité ou d'incompétence ${ }^{5}$.

En soi, il s'agit là d'une tentative de schématisation de réformes très valable, mais qui s'applique surtout au contexte américain. En effet, compte tenu des différences, selon les pays, des modes de sélection et de nomination, ou d'élection des juges, l'utilisation des normes similaires est limitée. Il n'en reste pas moins que les commissaires explicitent les principes sous-jacents aux normes et que, par conséquent, il est possible de procéder aux analyses comparatives à ce niveau.

3. Les réformes de l'application de la justice criminelle; le procès criminel et l'élaboration de la sentence

Au-delà des normes concernant les principaux agents de l'application de la justice criminelle, se situent celles de leur intervention lors de diverses étapes du processus judiciaire mais aussi de son déroulement en tant que tel.

Il peut paraître significatif, à cet égard, que dans la première partie qui traite du procès criminel on s'attache à décrire les relations entre les avocats, juges, agents de probation et autres services concernés, avec la presse. Ce qui frappe c'est l'importance qu'on confère au respect de la confidentialité et le souci de la réglementer.

Les auteurs du rapport réfèrent aux deux amendements de la Constitution américaine, le premier et le sixième, afin de mieux illustrer l'absence de contradiction entre les deux objectifs ; celui de protéger les droits de l'individu à une justice pleine et entière et celui d'assurer la liberté d'expression et d'information du public.

Par la suite, ils élaborent cependant des normes de confidentialité devant être respectées à chaque étape du procès, $\mathbf{y}$ compris celles relatives au jury, mais ne traitent nullement des garanties qui pourraient éventuellement être exigées des journalistes ou des Conseils supérieurs de la presse.

Le problème a été discuté au Québec à l'occasion des propositions visant à autoriser l'admission des représentants de

5. Pour le Québec, voir la nouvelle législation proposée par le ministre de la Justice, Marc-Anđré Bédard, relative à la création du Conseil de la magistrature. 
la presse aux audiences des tribunaux pour mineurs et il peut paraître décevant, pour un lecteur québécois, de ne trouver dans le rapport rien de novateur en la matière. D'une manière plus générale, les commissaires précisent que des discussions se poursuivent entre le Barreau, les juges, les autorités administratives concernées et les media d'information, mais ne traitent pas de résultats obtenus jusqu'à présent. On a l'impression, sur la base du texte du rapport, que tout se passe comme si la méfiance à l'égard de la viabilité des engagements qui pourraient être pris éventuellement par les journalistes, prédominait à un point tel qu'aucun examen objectif d'une pareille approche ne saurait être envisagé dans l'immédiat.

L'analyse des règles relatives au procès proprement dit commence par celles concernant la détention préventive et la libération sur cautionnement. Là encore on peut avouer une certaine déception. II apparait, en effet, que tout en critiquant largement les disparités et les injustices qu'entraîne la mise en liberté provisoire conditionnée par un dépôt de garantie financière, les commissaires n'insistent pas suffisamment sur les possibilités de libération sur parole, telles qu'utilisées au Canada, par exemple, depuis la promulgation du bill Omnibus.

Tout en mentionnant certains programmes dont, entre autres, celui de Vera Foundation et du ministère de la Justice, qui date de 1964, ils indiquent plusieurs règles de conduite pouvant réduire sensiblement le nombre de prévenus incarcérés avant procès. Ils insistent notamment sur la nécessité pour les juges d'émettre des sommations de préférence aux mandats d'arrestation et cela dans tous les cas où cela est possible, comme sur les avantages des méthodes consistant à confier le prévenu à un organisme ou à un agent de probation, à charge pour eux de s'assurer qu'il comparaîtra à son procès.

En d'autres termes, les commissaires proposent une utilisation plus large du pouvoir dont disposent les juges, et d'une manière plus générale, on a l'impression qu'ils considèrent que, contrairement aux époques précédentes, il n'est pas fondamental désormais de promouvoir des réformes législatives, mais plutôt de structurer et d'assouplir le fonctionnement de l'appareil de la justice. À cet égard, il s'agit d'une approche relativement originale dont on ne saurait méconnaître l'importance empirique. 
Cette remarque s'applique tout aussi bien au problème de détention préventive qu'à celui de certaines pratiques dont, entre autres, celle des procédures accélérées (speedy trial). Les commissaires justifient leur existence par la surcharge des cours et ne réclament pas leur suppression mais indiquent plusieurs normes et règles qui doivent être rigoureusement observées, selon eux, pour éliminer les possibilités des injustices et des erreurs.

En d'autres termes, autant les règles relatives à la conduite et aux objectifs devant être atteints par des agents de la justice visent surtout à l'accroissement de l'efficacité et de la valeur professionnelle de leur action, autant celles qui ont trait à l'evolution du processus de l'application de la justice, semblent destinées principalement à protéger et à aider l'individu trouvé coupable d'un délit. Ce souci apparaît à travers les normes proposées pour éviter au plus grand nombre la détention préventive comme au niveau de celles relatives aux sentences alternatives à la peine d'emprisonnement.

Dès le départ, les commissaires insistent sur le fait que le législateur doit se contenter de mettre à la disposition des magistrats un large éventail des mesures qu'ils peuvent appliquer sans être obligés, pour autant, de respecter des exceptions autres que celles concernant des criminels condamnés à la peine capitale, et sans être tributaires des limites rigides des peines minimale et maximale.

En effet, l'individualisation des sentences exige qu'on tienne compte des capacités et du potentiel de réinsertion sociale de chaque condamné.

C'est ainsi que les commissaires proposent qu'on établisse un maximum de cinq ans pour la plupart des délits graves et que seuls les criminels d'habitude, ou des récidivistes ayant des dossiers particulièrement chargés, puissent être condamnés à des périodes se situant au-delà de cette limite.

À l'intérieur de ce cadre, on traite de cinq types de sentences ; la probation, la détention intermittente, l'amende et le remboursement des victimes, la peine privative de liberté et le traitement obligatoire.

Dans leurs normes, les commissaires insistent sur l'objectif visant à laisser le maximum de latitude aux juges en ce qui a 
trait à l'éligibilité des condamnés à des mesures ne prévoyant pas de peine privative de liberté. Ils établissent cependant des propositions des règles limitant la durée des périodes prévues par les sentences de probation, ou de détention intermittente, ainsi que celles relatives aux modes et aux délais de paiement d'amende ou de dédommagement.

Il convient aussi de souligner l'importance donnée aux rapports présentenciels et aux critères des prononcés des sentences. Le Comité suggère, entre autres, à ce propos, la création d'un institut où les juges seraient invités à discuter de ces critères, à suivre des cours d'information et à visiter les diverses institutions et services disponibles où les condamnés sont placés et qui ont la charge de superviser leur conduite. Les commissaires ajoutent que les juges devraient être en mesure, en outre, de suivre l'évolution des individus condamnés par eux, afin de pouvoir se rendre mieux compte des résultats obtenus à la suite de l'exécution d'un type de sentence comparativement à un autre.

Parallèlement, les auteurs du rapport insistent sur l'obligation pour l'avocat de la défense, de s'assurer que le délinquant dispose d'une aide et d'une assistance sociale dont il a besoin et soulignent la nécessité de créer des services à cet effet.

En somme, globalement, les commissaires semblent donner beaucoup d'importance à la marge d'autonomie que le législateur doit laisser aux juges, tout en insistant sur le perfectionnement des magistrats qui, selon eux, doivent être plus au fait de l'évolution et des progrès réalisés par les sciences humaines que ce n'est le cas actuellement.

Au niveau de certaines règles spécifiques, on trouve cependant des précisions qui délimitent le cadre de cette approche. Pour ne citer qu'un seul exemple, les commissaires considèrent qu'en ce qui a trait aux amendes, le législateur doit énumérer les catégories de délits pour lesquels elles peuvent être imposées ainsi que le maximum du montant exigible. À l'opposé, il incombera au tribunal de fixer les modalités de versements de l'amende, calculées selon les capacités de paiement du condamné, compte tenu des charges et des obligations auxquelles il doit, par ailleurs, faire face.

Les commissaires proposent, en outre, qu'on inclut dans le Code criminel les conditions selon lesquelles le montant de 
l'amende devra être calculé afin qu'elles puissent constituer une sorte de guide de conduite pour les juges. Finalement, ils stipulent que les tribunaux ne doivent pas être autorisés à imposer des équivalences en nombre de jours d'emprisonnement telles que " trente dollars ou trente jours de détention $\gg$, comme cela se pratique pour le moment aux Etats-Unis comme au Québec ${ }^{\circ}$.

A travers les normes, les commissaires proposent donc au législateur la création d'un cadre, parfois très large et parfois très spécifique, imposant aux juges une diversification et une individualisation des sentences et aux administrateurs l'obligation de fournir des services sociaux de support.

En ce qui a trait à la police, les normes étaient surtout orientées vers une interprétation analytique de son rôle vers un classement de ses objectifs et vers les précisions des limites de ses pouvoirs. À l'opposé, le processus judiciaire est traité de façon à indiquer le cadre de son fonctionnement, l'importance de ses responsabilités et les réformes qui doivent être entreprises.

Ce qui prédomine comme approche c'est le souci d'une formulation autorisant une application immédiate. Le rapport est comparable en cela à certains travaux effectués au Canada par la Commission de réforme de droit.

En ce qui a trait à la philosophie sous-jacente aux normes proposées, elle refiète, selon nous, les préoccupations de l'heure. Les commissaires insistent sur l'urgence d'obtenir une plus grande efficacité et un sens accru des responsabilités professionnelles de la part de ceux qui sont chargés d'appliquer la justice criminelle. Ils justifient cette exigence fondamentale par les nécessités d'assurer la sécurité et l'ordre public, mais aussi, sinon surtout, par les impératifs de la réinsertion sociale des délinquants et des marginaux.

Il est assez ironique de constater, écrivent les commissaires, que le processus de l'établissement de culpabilité est entouré de multiples précautions et que, selon les statistiques, on ne remet en cause que 10 à $20 \%$ de cas où la preuve de la culpabilité a été faite et retenue par le tribunal. A l'opposé, les précautions qui

6. Pour détails, voir le rapport sur l'emprisonnement à défaut de paiement d'amende. André Campeau, Etude sur les modalités et les conséquences de l'emprisonnement à défaut de paiement d'amende, sous la direction de A. Parizeau, Montréal, Québec, 1975, 66 p. 
entourent la prise des décisions relatives à la punition imposée sont en fait minimes, bien que la réinsertion sociale du délinquant en dépende pour une très large part. Car on sait que les sentences excessives, comme celles dont la rationalité n'est pas dictée par la situation du condamné, mais par les habitudes acquises, minent le respect de la loi et vont à l'encontre de tous les autres objectifs qu'on veut atteindre.

Aux États-Unis, la procédure d'appel s'accompagne d'une révision particulière des sentences qui existe dans les Etats du Connecticut, du Maine, du Maryland et du Massachusetts où siègent, à cet effet, des comités spéciaux de juges. Il s'agit là d'une procédure qui existe en Grande-Bretagne depuis 1907 et qui constitue une protection des condamnés contre des excès et des illogismes de l'appareil judiciaire.

Les commissaires consacrent beaucoup d'attention aux normes devant régir selon eux les diverses procédures de révision de sentences et d'appel et insistent longuement sur la nécessité de les mettre en pratique.

Il n'est pas inutile, nous semble-t-il, d'établir à cet égard un parallèle avec le système canadien. En effet, la Commission des libérations conditionnelles, organisme formé des citoyens qui ne sont pas nécessairement des juristes, reçoit les demandes présentées par tous les prisonniers qui désirent le faire. Sans que cela soit précisé explicitement, il s'agit là d'une forme de révision des sentences d'autant plus intéressante qu'elle n'impose pas de dépenses aux concernés. Par ailleurs, le règlement selon lequel tout prisonnier peut écrire directement à la Commission, sans avoir pour cela une autorisation du directeur de la maison de détention, constitue une garantie fondamentale de ses droits.

Certes les pouvoirs de la Commission des libérations conditionnelles sont limités à la mise en liberté sous conditions. Cela signifie qu'elle ne peut pas renverser la décision judiciaire en ce qui a trait à la durée de la peine et libérer complètement un prisonnier de toute obligation, mais qu'elle est en mesure de raccourcir sensiblement la période qu'il doit, selon la sentence, passer en détention.

En ce qui a trait à la procédure d'appel, proprement dite, elle est relativement côteuse non seulement au Canada, mais dans la majorité des pays occidentaux. A notre connaissance, 
jusqu'à présent plusieurs tentatives de suppression de frais de procédure ont été proposées (comme c'est également le cas pour les Commissaires qui mentionnent cet aspect dans leurs normes), mais sans succès. Il s'agit là moins d'un refus d'une éventuelle perte des revenus pour l'appareil de la justice que d'un frein destiné à empêcher l'accroissement indû des causes en appel et les retards judiciaires que cela occasionnerait fatalement. La Commission d'enquête sur l'administration de la justice en matière criminelle et pénale au Québec ${ }^{7}$ a étudié la possibilité d'instaurer un système selon lequel la procédure d'appel serait gratuite dans un premier temps, mais l'appelant serait tenu à rembourser tous les frais si sa demande devait être rejetée par une cour supérieure. Pour des raisons techniques, la tentative de formuler une proposition de réformes allant dans ce sens a été abandonnée cependant et ne figure pas dans les recommandations de la Commission.

\section{Conclusion}

D'une manière globale, on ne peut pas ne pas être impressionné par le travail accompli par le Comité du Barreau américain et par l'effort de planification et de normalisation qu'il représente. Il s'agit là d'un document de base pouvant être utilisé dans d'autres contextes nationaux, autant pour étudier les structures existantes que pour planifier leur amélioration. Il n'en reste pas moins que la tentative de réformer l'application de la loi dans le domaine de la justice criminelle, grâce à l'élaboration des normes claires, précises et très concrètes, ne saurait être considérée, à notre humble avis, comme une solution, mais uniquement comme un outil de travail.

En effet, les lacunes du système ne sont pas inhérentes uniquement à l'absence, ou à l'insuffisance des guides, ou des cadres d'action, mais à un ensemble de facteurs fort complexes. Il s'agit là tout aussi bien des conséquences d'une insuffisance chronique des ressources que des difficultés de recrutement des professionnels de valeur, ou encore des problèmes de collaboration qui apparaissent entre les juges, les travailleurs sociaux et les spécialistes de diverses disciplines tels entre autres les psychiatres

7. Québec, Commission d'enquête sur l'administration de la justice en matière criminelle et pénale au Québec, la Société face au crime, Editeur officiel du Québec, 1968. 
et les psychologues. Aussi longtemps que la justice criminelle était axée sur l'élaboration de la preuve de culpabilité et sur l'imposition d'une peine de prison, le rôle des cours était surchargé dans les grandes agglomérations urbaines, mais on ne remettait pas constamment en cause leur mode de fonctionnement et d'application de la justice. Désormais, la diversification des sentences, les nombreuses tentatives d'individualiser les mesures prises, en fonction non pas de la catégorie du délit commis, mais de la personnalité du délinquant, exigent une approche multidisciplinaire.

A l'intérieur des cours, des juristes doivent côtoyer dès lors un personnel qui n'a pas la même formation qu'eux et qui s'insurge contre le langage hermétique de la justice. Pris par surprise, les administrateurs des tribunaux sont confrontés avec des obligations nouvelles, dont celle de tenir à jour des statistiques des prévenus et des condamnés, a suscité, à elle seule, des difficultés quasi insurmontables. On demande à l'appareil de la justice, traditionnaliste par définition, de secouer son «folklore » et d'utiliser des ordinateurs, non seulement pour recenser les diverses catégories des personnes inculpées, jugées et condamnées, mais encore pour évaluer l'efficacité du travail accompli par son personnel. Dans un tel contexte, l'introduction des normes ne peut être valable que dans la mesure où on disposera de gens prêts à les appliquer. Ce qui signifie qu'on doit tenir compte de cet objectif au niveau de la formation de ceux qui vont prendre la relève au cours des années à venir.

L'esprit qui a présidé à la rédaction des normes apparaît, dans un tel contexte, fort intéressant, mais leur mise en pratique ne saurait apporter des changements en profondeur, selon nous, aussi longtemps qu'on ne parviendra pas à donner une formation adéquatement complétée aux juristes et aux travailleurs sociaux chargés de les appliquer. 


\section{BIBLIOGRAPHIE}

American Bar Association Project on Standards for Criminal Justice, The Urban Police Function, American Bar Association, Approved by the House of Delegates, June 1973, 303 p.

American Bar Association Project on Standards for Criminal Justice, Discovery and Procedure Before Trial, American Bar Association, Approved by the House of Delegates, October 1970, 167 p.

American Bar Association Project on Standards for Criminal Justice, The Prosecution Function and the Defense Function, American Bar Association, Approved by the House of Delegates, March 1971, 327 p.

American Bar Association Project on Standards for Criminal Justice, Providing Defense Services, American Bar Asseciation, Approved by the House of Delegates, June 1967, 85 p.

American Bar Association Project on Standards for Criminal Justice, Pretrial Release, American Bar Association, Approved by the House of Delegates, September 1968, $88 \mathrm{p}$.

American Bar Association Project on Standards for Criminal Justice, Fair Trial and Free Press, American Bar Association, Approved by the House of Delegates, March 1968, 265 p.

American Bar Association Project on Standards for Criminal Justice, Speedy Trial, American Bar Association, Approved by the House of Delegates, May 1967, 56 p.

American Bar Association Project on Standards for Criminal Justice, Joinder and Severance, American Bar Association, Approved by the House of Delegates, September 1968, 68 p.

American Bar Association Project on Standards for Criminal Justice, Pleas of Guilty, American Bar Association, Approved by the House of Delegates, March 1968, 78 p.

American Bar Association Project on Standards for Criminal Justice, Sentencing Alternatives and Procedures, American Bar Association, Approved by the House of Delegates, September 1968, 345 p.

American Bar Association Project on Standards for Criminal Justice, Trial by Jury, American Bar Association, Approved by the House of Delegates, September 1968, $180 \mathrm{p}$.

American Bar Association Project on Standards for Criminal Justice, Appellate Review of Sentences, American Bar Association, Approved by the House of Delegates, March 1968, $160 \mathrm{p}$.

American Bar Association Project on Standards for Criminal Justice, Probation, American Bar Association, Approved by the House of Delegates, February 1970,110 p.

American Bar Association Project on Standards for Criminal Justice, Post-Conviction Remedies, American Bar Association, Approved by the House of Delegates, January 1967, 123 p. 